\title{
FORMING OF DISCRETE MECHANICAL ASSEMBLY PRODUCTION PROGRAM
}

A. V. Panyukov, South Ural State University, Chelyabinsk, Russian Federation, paniukovav@susu.ac.ru

V. A. Teleghin, South Ural State University, Miass, Russian Federation, vadimt_82@mail.ru

Searching of the optimal production program methods are observed. The aggregated mathematical model and the model with the alternative technological rotes and the bottleneck problem are proposed. In addition, the procedure of deriving of new production established standards based on accumulation statistics is proposed. Ways of flexible automation of production problem search processes for enterprises are denoted.

Keywords: production program, transportation problem, factory-assembled problem, working center, pattern of production, established standards, guidelines.

\section{Criteria of Production Program Optimality}

The criterion of an optimality is maximizing of marginal profit. But it is necessary to take into account the main limiting factors: demand and production capacity. The marginal profit is determined by a price and a prime cost of product. The price is determined by an official price list of company or by conditions of a contract with customer. The demand is determined by forecast, by volume of supplies to customer recorded in the contracts and orders. The assortment of products is made by a product range, product versions, a product quality and a packing. The combination of the product assortment, the contract, the sales condition, the demand, and the price are different for every sales condition. The prime price depends on the product range. The marginal profit is different for different sales conditions [1], [8]. The main optimality criterion that depends on a production volume, and a product assortment is a marginal profit. Using a marginal profit the following indices can be enumerated:

(1) a marginal profit per a product unit;

(2) a marginal profit per unit of a general product labour intensiveness;

(3) a marginal profit per unit of a labour intensiveness of the bottle-neck in the route of production;

(4) a ratio of a marginal profit per unit of general product labour intensiveness to its general labour intensiveness [7].

\section{Optimal Production Program Forming}

It is necessary to create the order of forming of the production program to evaluate the effectiveness of using of the production facilities and of the product assortment management. Basically, three main stages of drawing of the production program can be marked out:

(1) evaluating of a labour intensiveness of the production program and comparing it with the available one; 
(2) calculating of the marginal profit by the kinds of products;

(3) analyzing of the marginal profit and a labour intensiveness.

Let us dwell upon the enumerated stages of forming of the production program. The labour intensiveness of the production program is calculated on the basis of a sale plan for forthcoming period of planning taking into account the available remaining stock of ready product and the minimum reserve stocks. The production program is converted into labour intensiveness units then the number of units to be produced has been planned. The time needed to make the product is calculated on the basis of engineering and design information.

Next step is comparing the planned demand for labour resources and the maximum available working time that includes overtime and days off. This allows to evaluate the ability of a company to fulfill the plan.

The production program is adjusted on the basis of such an index as a marginal profit. In other words, products with the lowest marginal profit per unit of the labour intensiveness are removed from the production program.

\section{Task of Optimizing of Production Program Considering the Alternative Production Routes and the "Bottle-Neck"}

Let us consider the situation at an enterprise with the machining process type of production that has a "bottle-neck" that is a section with the lowest output consisting of equipment of different productivity. The "bottle-neck" consists of alternative working centers. A working center is a section, a workplace. Thus, it is necessary to take into account only the "bottle-neck" under modeling the limitation by the production capacity. Let $m$ be the number of working centers, $J=\{1,2, \ldots, m\}$ be a set of alternative working centers. Let $n$ be the number of sales conditions, $I=\{1,2, \ldots, n\}$ be a set of sale conditions, $p_{i}$ be the marginal profit and $d_{i}$ be the demand on the $i$-th sale condition.

The mathematical model of optimizing of a production program by the "bottle-neck" considering alternative working centers can look like the following

$$
\begin{gathered}
\sum_{i=1}^{m} \sum_{j=1}^{n} p_{i j} x_{i j} \rightarrow \max _{x}, \\
\sum_{j=1}^{n} x_{i j}=d_{i}, i=1,2, \ldots, n, \\
\sum_{i=1}^{m} \lambda_{i j} x_{i j} \leq b_{j}, j=1,2, \ldots, m, \\
x_{i j} \geq 0, \quad i=1,2, \ldots, n, j=1,2, \ldots, m,
\end{gathered}
$$

where $x_{i j}$ is the quantum of output sold by $i$-th sale condition and produced at $j$-th working center, $p_{i j}$ is the marginal profit from the sales of products by $i$-th sale condition and produced at $j$-th working center, $d_{i}$ is the demand for products by $i$-th sale condition, $\lambda_{i j}$ is the coefficient or index of machining labour intensiveness for $i$-th products at $j$-th working center, $b_{j}$ is the availability or the fund of working time for $j$-th working center [6]. 
This problem belongs to the type of distributive ones of linear programming. "Distribution problems" is a well-known class of fundamental combinatorial optimization problems, which are much more difficulty to solve than assignment problems [2]. Under certain conditions it can be transformed into a transportation problem. To this end, it is necessary to use a presentation $\lambda_{i j}=\alpha_{i} \beta_{j}$, where $\alpha_{i}$ is the relative labour intensiveness of the machining of $i$-th product, $\beta_{j}$ is the given productivity of $j$-th working center [5].

The transformations given below determine the method of reducing of distributive problem (1)-(4) to matrix transportation problem.

$$
\begin{array}{r}
(\forall j=1,2, \ldots, m) \\
\left(\sum_{i=1}^{m} \lambda_{i j} x_{i j} \leq b_{j} \Leftrightarrow \sum_{i=1}^{m} \alpha_{i} \beta_{j} x_{i j} \leq b_{j} \Leftrightarrow \sum_{i=1}^{m} \alpha_{i} x_{i j} \leq \frac{b_{j}}{\beta_{j}} \Leftrightarrow \sum_{i=1}^{m} y_{i j} \leq \frac{b_{j}}{\beta_{j}}\right),
\end{array}
$$

where

$$
y_{i j}=\alpha_{i} x_{i j}, \quad i=1,2, \ldots, n, \quad j=1,2, \ldots, m
$$

is planned labour intensiveness of the works on the $i$-th product at the $j$-th working center. Passing over to variables $y_{i j}=\alpha_{i} x_{i j}$ in (1) and (4), we get

$$
\begin{gathered}
(\forall i=1,2, \ldots, n)\left(\sum_{j=1}^{n} x_{i j}=d_{i} \Leftrightarrow \sum_{j=1}^{n} y_{i j}=d_{i} \alpha_{i}\right), \\
\left(\sum_{i=1}^{m} \sum_{j=1}^{n} p_{i j} x_{i j} \rightarrow \max _{x}\right) \Leftrightarrow\left(\sum_{i=1}^{m} \sum_{j=1}^{n} p_{i j} \alpha_{i} y_{i j} \rightarrow \max _{y}\right) .
\end{gathered}
$$

Thus, distributive sum (1) - (4) is equivalent to the sum:

$$
\begin{gathered}
\sum_{i=1}^{m} \sum_{j=1}^{n} p_{i j} \alpha_{i} y_{i j} \rightarrow \max _{y} \\
(\forall i=1,2, \ldots, n)\left(\sum_{j=1}^{n} y_{i j}=d_{i} \alpha_{i}\right), \\
(\forall j=1,2, \ldots, m)\left(\sum_{i=1}^{m} y_{i j} \leq \frac{b_{j}}{\beta_{j}}\right), \\
(\forall i=1,2, \ldots, n, \forall j=1,2, \ldots, m)\left(y_{i j} \geq 0\right) .
\end{gathered}
$$

Problem (5) - (8) is known as the open matrix transportation problem. Using simple transformations problem $(5)-(8)$ can be reduced to a closed transportation problem.

The presented transportation problem can be solved using method of potentials, algorithm of disorder, direct or dual simplex-method. The development of modern mathematical supply of solving optimal flow problem, transportation problem began in 1970-th. The comparison of algorithms to these tasks solve, the experience of operation and the calculating experiments have shown that the direct simplex-method by far surpasses the other methods known [4]. 
Thus, the problem of optimizing of production program considering the "bottle-neck" and alternative working centers belongs to distributive problems of linear programming. To solve this problems, the method of reduction of distributive problem to a closed matrix transportation problem has been suggested. A modified simplex-method, a program tool to realize it, as well as integration modules with known corporate information systems have been suggested to solve the transportation problem. The using of the effective flow algorithms allows effectively solve the problem of building up the optimal production programs for the cases when

$$
\lambda_{i j}=\alpha_{i} \beta_{j}, i=1,2, \ldots, n, j=1,2, \ldots, m .
$$

The authors have suggested the method of determining the volume-calendar norms

$$
\alpha_{i}, \quad i=1,2, \ldots, n, \quad \beta_{j}, j=1,2, \ldots, m
$$

for new products on the basis of accumulated statistics $\lambda_{i j}, i=1,2, \ldots, n, j=1,2, \ldots, m$ on the products that are already being produced in [5], [6].

\section{Target Setting for Calendar Plan Standards}

The main elements of reference system for planned-economic calculations are the calendar-plan standards. They are used for planned calculation, yield of capital investments, production program. In practice, the rate of output and the labour intensiveness are found out using of a photo of working day, statistics of product machining by workplaces or by experts [5].

To reduce distributive problem $(1)-(4)$ to transportation problem $(5)-(8)$ it is necessary to factorize the coefficient or the index of machining labour intensiveness $\lambda_{i j}$ by the product of indices $\alpha_{i}$ and $\beta_{j}$ having given complexity of $i$-th product and given productivity of $j$-th working center correspondingly [6].

The determining of indices of the product relative labour intensiveness and the productivity of workplaces can be used:

(1) For the analytical calculation of the potential labour intensiveness and the rate of output at workplaces of a section where the product has not been machined yet, but is supposed to be machined.

(2) For the calculation of the planned prime cost without considering different piece-rates for production on the alternative technological routes.

(3) For the evaluation of the potential yield of capital investments from equipment, workplaces of alternative section for a comparative analysis of the use of different type of equipment, alternative technological routes.

Let us consider a method of finding of indices $\alpha_{i}$ and $\beta_{j}$ values by measured indices $\lambda_{i j}$. Note that

$$
\begin{aligned}
&\left(\lambda_{i j}=\frac{\alpha_{i}}{\beta_{j}}\right) \Leftrightarrow\left(\ln \lambda_{i j}=\ln \alpha_{i}-\ln \beta_{j}\right) \Leftrightarrow \\
&\left(a_{i j}=x_{i}-y_{j}, a_{i j}=\ln \lambda_{i j}, x_{i}=\ln \alpha_{i}, y_{j}=\ln \beta_{j}\right),
\end{aligned}
$$


therefore in further we consider the problem in terms $x_{i}, y_{j}$ using the technique of least modules

$$
\sum_{i \in I, j \in J}\left|x_{i}-y_{j}-a_{i j}\right| \rightarrow \min _{x, y}
$$

This problem is equivalent to linear programming problem

$$
\begin{gathered}
\sum_{i, j} w_{i j} \rightarrow \min _{x, y, w} \\
-w_{i j} \leq x_{i}-y_{j}-a_{i j} \leq w_{i j}, w_{i j} \geq 0, i \in I, j \in J,
\end{gathered}
$$

which in the standard form looks like

$$
\begin{gathered}
\sum_{i, j} w_{i j} \rightarrow \min _{x, y, w}, \\
(\forall i \in I, j \in J)\left(\begin{array}{l}
x_{i}-y_{j}+w_{i j} \geq a_{i j} \\
-x_{i}+y_{j}+w_{i j} \geq-a_{i j} \\
w_{i j} \geq 0
\end{array}\right) .
\end{gathered}
$$

The dual problem to problem (11)-(12) is

$$
\begin{gathered}
\sum_{i \in I, j \in J} a_{i j}\left(f_{i j}-f_{j i}\right) \rightarrow \max _{f}, \\
(\forall i \in I)\left(\sum_{j \in J} f_{i j}-\sum_{j \in J} f_{j i}=0\right), \\
(\forall j \in J)\left(\sum_{i \in I} f_{j i}-\sum_{i \in I} f_{i j}=0\right), \\
(\forall i \in I, \forall j \in J)\left(f_{i j}+f_{j i} \leq 1\right), \\
(\forall i \in I, \forall j \in J)\left(f_{i j}, f_{j i} \geq 0\right) .
\end{gathered}
$$

In the resulting sum let us make a transformation of variables

$$
g_{i j}=f_{i j}-f_{j i}+1, \quad h_{i j}=f_{i j}+f_{j i}, \quad i \in I, j \in J .
$$

After the transformation of variables we have matrix transportation problem

$$
\begin{gathered}
-|I| \cdot|J|+\sum_{i \in I, j \in J} a_{i j} g_{i j} \rightarrow \max _{g}, \\
(\forall i \in I)\left(\sum_{j \in J} g_{i j}=|J|\right), \\
(\forall j \in J)\left(-\sum_{i \in I} g_{i j}=-|I|\right), \\
(\forall i \in I, \forall j \in J)\left(0 \leq g_{i j} \leq 2\right),
\end{gathered}
$$


that can be solved using the method of potentials. This method along with solving of (19)-(22), finds solution to the corresponding dual problem

$$
\begin{gathered}
|J| \cdot \sum_{i \in I} r_{i}-|I| \cdot \sum_{j \in J} s_{j}+2 \cdot \sum_{i \in I, j \in J} t_{i j} \rightarrow \min _{r, s, t}, \\
(\forall i \in I, \forall j \in J)\left(r_{i}-s_{j}+t_{i j} \leq a_{i j}\right), \\
(\forall i \in I, \forall j \in J)\left(0 \leq t_{i j}\right) .
\end{gathered}
$$

Comparing system of limitations (11) - (12) with system of limitations $(22)-(24)$, it is easy to see that from the permissibility of solution $(r, s, t)$ to problem $(22)-(24)$ results the permissibility of solution

$$
(x=r, y=s, w=t)
$$

of problem $(11)-(12)$. Moreover, if $(r, s, t)$ is an optimal solution to problem 22) - (24), then

$$
(x=r, y=s, w=t)
$$

is an optimal solution to problem $(11)-(12)$, because the dual to them problems (13) (17) and (18) - (21) have the corresponding optimal solutions.

The foregoing makes it possible to suggest the following algorithm of calculating of the given product complexity and the given equipment productivity

A L G O R I T H M A

- INPUT:

- Product list $I$;

- Equipment list $J$

$-\Lambda=\left\{\lambda_{i j}: i \in I, j \in J\right\}$

- O U T P U T :

$-\left\{\alpha_{i}: i \in I\right\}$

$-\left\{\beta_{j}: j \in J\right\}$

- S T EP 1. Calculate matrix

$$
\mathrm{A}=\left\{a_{i j}=\ln \lambda_{i j}: i \in I, j \in J\right\}
$$

by matrix

$$
\Lambda=\left\{\lambda_{i j}: i \in I, j \in J\right\} .
$$

- S T E P 2. Find the optimal solutions to the pair of mutually dual problems (18) (21) and (22) - (24) for given I, J, A. Let it be g and (r,s,t) correspondingly.

- S T E P 3. For each $i \in I$ calculate $\alpha_{i}=\exp \left(r_{i}\right)$.

- STEP 4. For each $j \in J$ calculate $\beta_{j}=\exp \left(z_{j}\right)$.

- S T E P 5. Return $\left\{\alpha_{i}: i \in I\right\},\left\{\beta_{j}: j \in J\right\}$.

- End of Algorithm A 


\section{Conclusion}

In order to calculate an optimal production program at an enterprise with a discrete mechanical assembly production, the following data are necessary:

(1) the list of product range;

(2) the average or the minimal selling price on all contracts for the period of planning;

(3) the planned calculation of every product prime cost;

(4) the sale plan on the basis of confirmed and forecasted customer orders or a forecast of sales on the basis of market analysis adjusted according to the warehouse remainder and the insurance stock;

(5) the data on the number of working shifts for the period of planning on every work place of the concrete section;

(6) the list of the unambiguously used technological routes of the production for every product for the period of planning;

(7) the rates of output on every work place on per working shift from the used technological routes of the production.

The second and the third clauses are necessary to determine the criterion function. The result of this calculation will become an array of indices $p_{i j}$ of the marginal profit in the criterion function. The fourth clause determines the demand limitation. The planned value of sales in the context of the product range is index $d_{i}$. The limitations on the production capacities are determined by the data in clauses 5 to 7 . The number of working shifts on all work places determines index $b_{j}$. Index $\lambda_{i j}$ is the coefficient of labour intensiveness of the concrete section.

Having these data and using algorithm A1 suggested in this article, one can find the volume-calendar standards one after another. The determining of the indices of relative labour intensiveness of the product and the productivity of a work place and sections can be used: for the analytical calculation of the potential labour intensiveness and rate of output at a work place of a section at which the products have not been machined, but is only supposed to be machined; for the calculation of the planned prime cost without considering different piece-rates for the production on alternative work places; for the evaluation of the potential yield of capital investments from equipment, work places, the alternative section for a comparative analysis of the use of equipment of different types, alternative technological routes.

The modern corporate information systems do not contain algorithms for doing linear programming sums. Program tools for doing sums of this type are developed separately from the process of the development of known corporate information systems. Actually, the described tools are presented by software modules, components and even separate commercial programs. Authors have developed the class TRANSPORT [4] for doing sums of the transportation type in different definitions which can be compiled into a class library for common operating systems. Also, an integration module has been developed using technologies for WINDOWS such as: COM, Web-services and XML [7]. 


\section{References}

1. Chen,Z.-L. Integrated Production and Outbound Distribution Scheduling: Review and Extensions. Operations Research, 2010, vol. 58, no. 1, pp. 130-148.

2. Geismar H. N., Laporte G., Lei L., Sriskandarajah C. The Integrated Production and Transportation Scheduling Problem for a Product with a Short Lifespan. Journal on Computing, 2008, vol. 20, no. 1, pp. 21-33.

3. Pundoor G., Chen Z.-L. Scheduling a Production-Distribution System to Optimize the Tradeoff Between Delivery Tardiness and Distribution Cost. Naval Research Logistics, 2005, vol. 52, no. 6, pp. 571-589.

4. Panyukov, A.V., Teleghin, V.A. Software Engineering of the Flow Algorithms. Bulletin of the South Ural State University. Series "Mathematical Modelling, Programming 65 Computer Software", 2008, no. 27(127), issue 2, pp. 78-99. (in Russian)

5. Panyukov A.V., Teleghin V.A. Application of Flow Programming for Engineering of Established Standards Bases. VI International Methodological Conference "Improvement of IT Personnel Training at Direction "Applied Informatics" for Innovate Economics": Conference Proceedings. Moscow, Moscow State University of Economics, Statistics and Informatics Publ., 2010, p. 214. (in Russian)

6. Panyukov A.V., Teleghin V.A. Approach to Forming of the Production Program for Enterprises with Discrete Mechanoerecting Type of Production. Perm University Herald. Economy, 2011, issue 4(11), p. 74-83. (in Russian)

7. Teleghin V.A., Latipova A.T. Integration of Optimization Program Batch to "1C:Enterprise 8". 11th International Conference "New Information Technologies of Education. Development of Innovate Educational Enterprises with Technologies "1C" (2010, February, 1 - 2): Conference Proceedings. Moscow, "1C-Publishing", 2011, p. 515-519. (in Russian)

8. Uporov V.A. Assortment Optimization Strategy for the Industrial Enterprise. Journal of Russian Entrepreneurship, 2009, no. 11, issue 1(146), pp. 94-97.

Anatoly V. Panyukov, Doctor of Physico-Mathematical Sciences, Professor, Head of Department of Economic and Mathematical Methods and Statistics, South Ural State University, Chelyabinsk, Russian Federation, paniukovav@susu.ac.ru.

Vadim A. Teleghin, Professor assistant, Department of Mathematical Modelling and Management, South Ural State University, Miass, Russian Federation, vadimt_82@mail.ru.

Received October 8, 2014 